\title{
Investigating causal relationships between exposome and human longevity: a Mendelian randomization analysis
}

Shu-Yi Huang ${ }^{1 \dagger}$, Yu-Xiang Yang ${ }^{1 \dagger}$, Shi-Dong Chen ${ }^{1}$, Hong-Qi Li ${ }^{1}$, Xue-Qing Zhang ${ }^{1}$, Kevin Kuo ${ }^{1}$, Lan Tan², Lei Feng ${ }^{3}$, Qiang Dong ${ }^{1}$, Can Zhang ${ }^{4}$ and Jin-Tai Yu ${ }^{1 *}$

\begin{abstract}
Background: Environmental factors are associated with human longevity, but their specificity and causality remain mostly unclear. By integrating the innovative "exposome" concept developed in the field of environmental epidemiology, this study aims to determine the components of exposome causally linked to longevity using Mendelian randomization (MR) approach.

Methods: A total of 4587 environmental exposures extracting from 361,194 individuals from the UK biobank, in exogenous and endogenous domains of exposome were assessed. We examined the relationship between each environmental factor and two longevity outcomes (i.e., surviving to the 90th or 99th percentile age) from various cohorts of European ancestry. Significant results after false discovery rates correction underwent validation using an independent exposure dataset.

Results: Out of all the environmental exposures, eight age-related diseases and pathological conditions were causally associated with lower odds of longevity, including coronary atherosclerosis (odds ratio $=0.77,95 \%$ confidence interval $\left.[0.70,0.84], P=4.2 \times 10^{-8}\right)$, ischemic heart disease $(0.66,[0.51,0.87], P=0.0029)$, angina $(0.73$, $\left.[0.65,0.83], P=5.4 \times 10^{-7}\right)$, Alzheimer's disease $\left(0.80,[0.72,0.89], P=3.0 \times 10^{-5}\right)$, hypertension $(0.70,[0.64,0.77], P=$ $\left.4.5 \times 10^{-14}\right)$, type 2 diabetes $(0.88[0.80,0.96], P=0.004)$, high cholesterol $(0.81,[0.72,0.91], P=0.0003)$, and venous thromboembolism $(0.92,[0.87,0.97], P=0.0028)$. After adjusting for genetic correlation between different types of blood lipids, higher levels of low-density lipoprotein cholesterol $\left(0.72[0.64,0.80], \mathrm{P}=2.3 \times 10^{-9}\right)$ was associated with lower odds of longevity, while high-density lipoprotein cholesterol $(1.36[1.13,1.62], P=0.001)$ showed the opposite. Genetically predicted sitting/standing height was unrelated to longevity, while higher comparative height size at 10 was negatively associated with longevity. Greater body fat, especially the trunk fat mass, and never eat sugar or foods/drinks containing sugar were adversely associated with longevity, while education attainment showed the opposite.
\end{abstract}

\footnotetext{
* Correspondence: jintai_yu@fudan.edu.cn

†Shu-Yi Huang and Yu-Xiang Yang contributed equally to this work.

${ }^{1}$ Department of Neurology and Institute of Neurology, Huashan Hospital, State Key Laboratory of Medical Neurobiology and MOE Frontiers Center for Brain Science, Shanghai Medical College, Fudan University, 12th Wulumuqi Zhong Road, Shanghai 200040, China

Full list of author information is available at the end of the article
}

(c) The Author(s). 2021 Open Access This article is licensed under a Creative Commons Attribution 4.0 International License, which permits use, sharing, adaptation, distribution and reproduction in any medium or format, as long as you give appropriate credit to the original author(s) and the source, provide a link to the Creative Commons licence, and indicate if changes were made. The images or other third party material in this article are included in the article's Creative Commons licence, unless indicated otherwise in a credit line to the material. If material is not included in the article's Creative Commons licence and your intended use is not permitted by statutory regulation or exceeds the permitted use, you will need to obtain permission directly from the copyright holder. To view a copy of this licence, visit http://creativecommons.org/licenses/by/4.0/. The Creative Commons Public Domain Dedication waiver (http://creativecommons.org/publicdomain/zero/1.0/) applies to the data made available in this article, unless otherwise stated in a credit line to the data. 
Conclusions: The present study supports that some age-related diseases as well as education are causally related to longevity and highlights several new targets for achieving longevity, including management of venous thromboembolism, appropriate intake of sugar, and control of body fat. Our results warrant further studies to elucidate the underlying mechanisms of these reported causal associations.

Keywords: Longevity, Mendelian randomization, Exposome

\section{Background}

Longevity is defined as the length or duration of life or viability, typically refer to the age of death or survival beyond of $90-100$ years or older [1]. It is a heterogenous trait that is susceptible to genetic and environmental factors. Previous genome-wide association studies (GWASs) have revealed genetic loci associated with human longevity or parental lifespan [2, 3], while environmental factors, including socio-economic status, smoking, gender, and lifestyle, are considered determinants [1]. Observational studies have also featured the associations on various risk factors, where the predicted longevity could be significantly reduced by cardiovascular disease (CVD), diabetes, hypertension, and tobacco smoking $[4,5]$. However, due to the vulnerability to reverse causation and confounding bias, most of the epidemiological studies are insufficient to draw a definite conclusion on causality.

Mendelian randomization (MR) is an analytical approach that can overcome such limitations by using genetic variants as instrumental variables (IVs) to evaluate the causal effect of exposure on the outcome. Since genotypes are randomly allocated from parents to offspring [6], MR method is less likely to be affected by reverse causality and measurement errors in the absence of pleiotropy, making causal inference more feasible compared to conventional study designs. Although several MR analyses have demonstrated a subset of environmental factors that were causally associated with longevity [7-9], the exploration of causal exposures is still in a relatively primitive stage. However, by applying the "exposome" concept proposed in the field of environmental epidemiology, we are able, for the first time, to investigate the totality of environmental exposures that affect an individual from conception until death [10]. Using the MR approach, our study aims to construct the potential components of exposome that causally linked to longevity.

\section{Methods}

\section{Exposure data}

UK Biobank (UKB) is a large-scale and long-term biobank with information on both genetics and broad environmental exposures collected over 10 years (www. ukbiobank.ac.uk). Over 500,000 individuals aged 40-69 years were recruited from across the UK between 2006 and 2010. The exposome data used in our MR analysis were originally from the UK Biobank. GWAS summary statistics of 4587 environmental exposures were obtained from the Neale Lab (http://www.nealelab.is/uk-biobank), based on 361,194 participants [11]. Categorical exposures with cases $<250$ and duplicated exposures were excluded [12]. Exposures with less than three independent single nucleotide polymorphisms (SNPs) at $\mathrm{P}<5 \times$ $10^{-8}$ were also excluded (Fig. 1). Finally, a total of 704 exposures were included in primary analysis, and 663 exposures were included in secondary analysis. We classified all these available exposures into three main domains: endogenous, exogenous individual and exogenous macro-level [13]. Exposures in each domain were then classified into different categories, mainly according to information in UKB.

\section{Outcome data}

We used two summary statistics from the largest metaanalysis of human longevity GWAS of European ancestry [3]. Longevity was defined as two dichotomous phenotypes [3]. Cases were individuals who lived beyond the 90th $(\mathrm{N}=11,262)$ or 99th $(\mathrm{N}=3484)$ percentile. Controls $(\mathrm{N}=25,483)$ were individuals who died at or before the age at the 60th percentile or whose age at the last follow-up visit was at or before the 60th percentile age. To mitigate the heterogeneity, the cohort-specific life tables for the country, sex, and birth, are used to identify the age threshold for cases and controls in the original GWAS [3]. Hence, the number of selected cases and controls is independent of the study population used. The 90th percentile longevity data was used in the primary analysis because of the larger sample size, while 99th percentile data was used as secondary analysis. The mean age of 90th percentile cases was 97 years, ranging from 87 to 122. The mean age of 99th percentile cases was 101 years, ranging from 90 to 122 . The mean age of the control group was 55 years, ranging from 0 to 88 . All participants provided written informed consent in original GWAS [3].

\section{Two-sample MR design}

We inferred causal relationships between each environmental exposure and longevity using two-sample MR, in which the selections of IVs are based on GWAS summary statistics generated from different, non-overlapping samples. To obtain unbiased estimates of the causal effects, MR analysis rests on three assumptions [6]: (i) the 


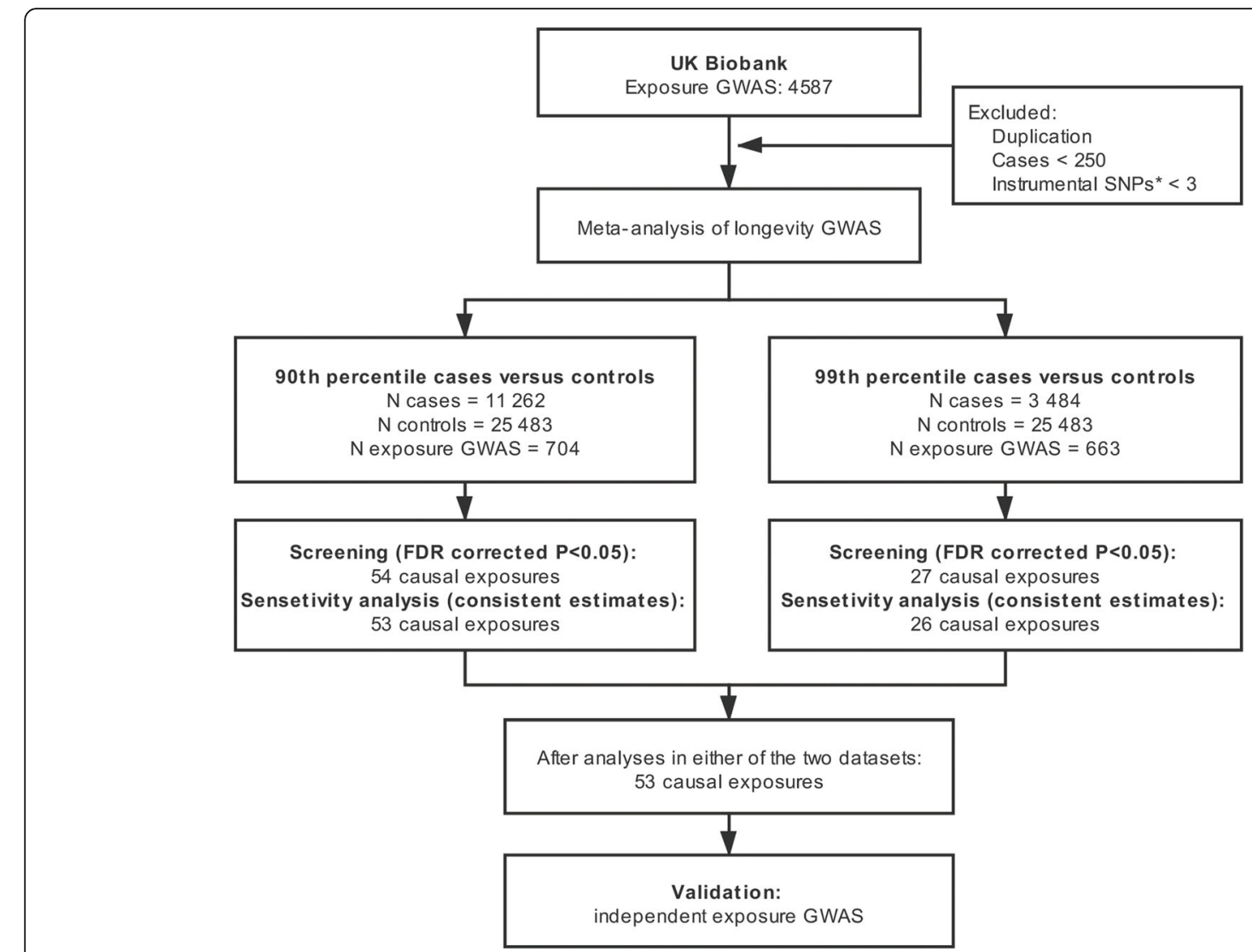

Fig. 1 A flow diagram of the study design and analysis process. FDR, false discovery rates; GWAS, genome-wide association study; N, number or sample size; SNPs, single nucleotide polymorphisms

genetic variants are associated with the exposure, (ii) the genetic variants are independent of confounders of the risk factor-outcome association, and (iii) the genetic variants influence the outcome only through the exposure.

\section{Selection of instrumental variables}

For each exposure, single nucleotide polymorphisms (SNPs) associated at P-value $<5 \times 10^{-8}$ with a minor allele frequency greater than 0.01 were considered potential instruments. We used MR-Base (http://www.mrbase. org) to select independent SNPs at a linkage disequilibrium threshold of $r^{2}<0.001$, and retained SNPs with the strongest effect on the associated trait. For palindromic SNPs, we aligned strands using allele frequency and discarded palindromic $\mathrm{SNP}(\mathrm{s})$ that had minor allele frequency above 0.42 . Then, exposure-outcome datasets were harmonized. We have considered the palindromic SNPs and checked original datasets to avoid reverse effects.

We computed the F-statistic of each exposure to judge the strength of IVs. The bias from weak instruments depends on the strength of the instrument through the F-statistic, which is related to the proportion of variance in the phenotype explained by IVs $\left(R^{2}\right)$, sample size (n) and number of instruments $(\mathrm{k})$ by the formula $\mathrm{F}=\left(\frac{n-k-1}{k}\right.$ $\left(\frac{R^{2}}{1-R^{2}}\right)$ [14]. Typically, a strong instrument was defined as an F-statistic $>10$ [14]. We estimated the statistical power with a false positive rate $\alpha=0.05$ using $\mathrm{R}$ code provided by Burgess $S$ [15]. Details of the genetic instruments were presented in Additional file 1: Table S1.

\section{Statistical analysis}

We used the inverse variance weighted (IVW) method as our principal MR analytical approach. This method will return an unbiased estimate in the absence of horizontal pleiotropy or when horizontal pleiotropy is balanced. Results are presented as odds ratio (OR) per standard deviation (SD) increase in genetically determined metabolites on $\mathrm{AD}$ for the outcome was dichotomous. For the Neal lab GWAS data using a linear model (rather than a logistic model) when analyzing case-control traits, thus, we applied a transformation according to the manual of BOLT_LMM (https:// alkesgroup.broadinstitute.org/BOLT-LMM/downloads/ BOLT-LMM_v2.3.4_manual.pdf) in order to convert SNP effect estimates ("betas") on the quantitative scale to traditional ORs. This approximate transformation is 
$\log$ OR $=\beta /(\mu \times(1-\mu))$, where $\mu=$ case fraction. Standard errors of SNP effect size estimates are also be divided by $(\mu \times(1-\mu))$ when applying that transformation to obtain $\log$ ORs.

Sensitivity analyses were conducted using weighted median [16], MR-Egger regression [17], and Mendelian randomization pleiotropy residual sum and outlier (MRPRESSO) [18]. These methods hold different assumptions at the costs of reduced statistical power. The weighted median allows for $50 \%$ of the IVs to be invalid or present pleiotropy [16]. MR-Egger regression allows > $50 \%$ of the variants to be invalid [17]. Heterogeneity in the IVW estimates was examined by Cochran's $Q$ test. Furthermore, MR-Egger intercept and MR-PRESSO global test were used to check for the presence of pleiotropy. In the case of horizontal pleiotropy, MR-PRES $\mathrm{SO}$ outlier test compares the observed and expected distributions of the tested variants to identify outlier variants. If significant outliers $(\mathrm{P}<0.05)$ are detected, they were removed from the analysis to return an unbiased causal estimate [18].

To correct for multiple comparisons, we applied false discovery rates (FDR) correction in IVW. An FDR corrected $\mathrm{P}$-value $<0.05$ was considered significant, and an unadjusted $\mathrm{P}$-value $<0.05$ was considered the evidence of a suggestive association. The significant traits with consistent point estimates across sensitivity analyses and IVW estimates were selected in the screening phase as the most robust causal exposures. Analyses were conducted using R version 3.6.3, with the MR analysis performed using the “TwoSampleMR" package version 0.5.2 [19].

\section{Validation}

For those identified significant exposures, we used nonUKB GWAS to validate our MR results. A total of 20 independent GWAS data were publicly available as part of the MRbase package [19]. If more than one GWAS were available for a given trait, an optimal one was selected based on large sample size, sufficient available SNPs, both sexes, and European or mixed descent. Details of independent exposure GWAS were presented in Additional file 1: Table S2. For each trait in the validation, IVs were constructed starting from all SNPs with $\mathrm{P}<5 \times 10^{-8}$. In validation analysis, the IVs of low-density lipoprotein cholesterol (LDL-C), high-density lipoprotein cholesterol (HDL-C), and triglycerides are partly overlapped [20]. Thus, we used multivariable MR to adjust for the genetic correlation [21]. Validation analyses were not conducted for those significant exposures without eligible data.

\section{Results}

\section{Screening results}

Of all analyzed exposures, 110 exposures and 73 exposures showed associations with longevity at $\mathrm{P}<0.05$ in the primary analysis and secondary analysis, respectively. We found that 53 exposures showed significant associations with either or both 90th and 99th percentile longevity after FDR correction and sensitivity analysis (Fig. 1). Of the 53 screening exposures, sensitivity analysis showed consistent point estimates with IVW in primary stage (Table 1). These exposures were classified into eight categories, including disease, physical measures, family history, medication, early life factors, education, lifestyle, and diet (Fig. 2, and Additional file 1: Table S3-S5). The list of the SNPs used as IVs for each screening exposure was presented in Additional file 1: Table S6. MR analyses were repeated using non-UKB exposure datasets (Fig. 3, and Additional file 1: Table S7-S9). A list of overview results in the present study is showed in Additional file 1: Table S10. All the significant and suggestive causal exposures from two longevity datasets are presented in Additional file 1: Table S11-S12. MR results of all traits are presented in Additional file 2: Table S13-S14.

Among reported exposures in Fig. 2, forty-two traits were associated with both 90th and 99th percentile survival longevity outcomes. In the disease category, diseases of circulatory system $\left(\mathrm{OR}_{90}=0.43[0.32,0.59], \mathrm{P}_{90}\right.$ $=1.0 \times 10^{-7}$ ) were causally associated with lower odds of 90th and 99th percentile longevity. We observed that ischemic heart disease $\left(\mathrm{OR}_{90}=0.66\right.$ [0.51, 0.87], $\mathrm{P}_{90}=$ $0.0029)$ was causally linked to both two longevity outcomes. MR-PRESSO global test and Q test showed substantial pleiotropy between the SNPs used as IVs for the two exposures $(\mathrm{P}<0.05$; Table 2$)$. However, after removing potential outlying SNPs, the corrected MR-PRES SO results are still significant. For other heart diseaserelated traits, coronary atherosclerosis $\left(\mathrm{OR}_{90}=0.77\right.$ $\left.[0.70,0.84], \mathrm{P}_{90}=4.2 \times 10^{-8}\right)$, cardiac arrhythmias with chronic obstructive pulmonary diseases $\left(\mathrm{OR}_{90}=0.86\right.$ $\left.[0.81,0.92], \mathrm{P}_{90}=1.9 \times 10^{-5}\right)$, self-reported angina $\left(\mathrm{OR}_{90}\right.$ $\left.=0.72[0.64,0.82], \mathrm{P}_{90}=2.3 \times 10^{-7}\right)$, and diagnosed angina $\left(\mathrm{OR}_{90}=0.73[0.65,0.83], \mathrm{P}_{90}=5.4 \times 10^{-5}\right)$ showed association with lower odds of 90th and 99th percentile longevity, while no vascular/heart problems $\left(\mathrm{OR}_{90}=\right.$ $1.55[1.41,1.71], \mathrm{P}_{90}=1.9 \times 10^{-19}$ ) showed the opposite. Pleiotropy tests were non-significant except for no vascular/heart problems (MR-Egger intercept $\mathrm{P}=0.0002$; global test $\mathrm{P}=0.001)$. Nevertheless, the corrected MR-PRESSO result was still significant after removing outliers.

Regarding blood pressure related traits, genetically predicted self-reported hypertension $\left(\mathrm{OR}_{90}=0.68[0.62\right.$, 0.74], $\left.\mathrm{P}_{90}=9.4 \times 10^{-17}\right)$ and diagnosed hypertension $\left(\mathrm{OR}_{90}=0.70[0.64,0.77] \mathrm{P}_{90}=4.5 \times 10^{-14}\right)$ were significantly associated with higher odds of both two longevity outcomes, with no outlying genetic variant identified. Quantitative increase of systolic blood pressure (SBP; $\left.\mathrm{OR}_{90}=0.55[0.44,0.68], \mathrm{P}_{90}=3.9 \times 10^{-8}\right)$ and diastolic blood pressure (DBP; $0.56[0.46,0.69], \mathrm{P}_{90}=3.4 \times 10^{-8}$ ) 


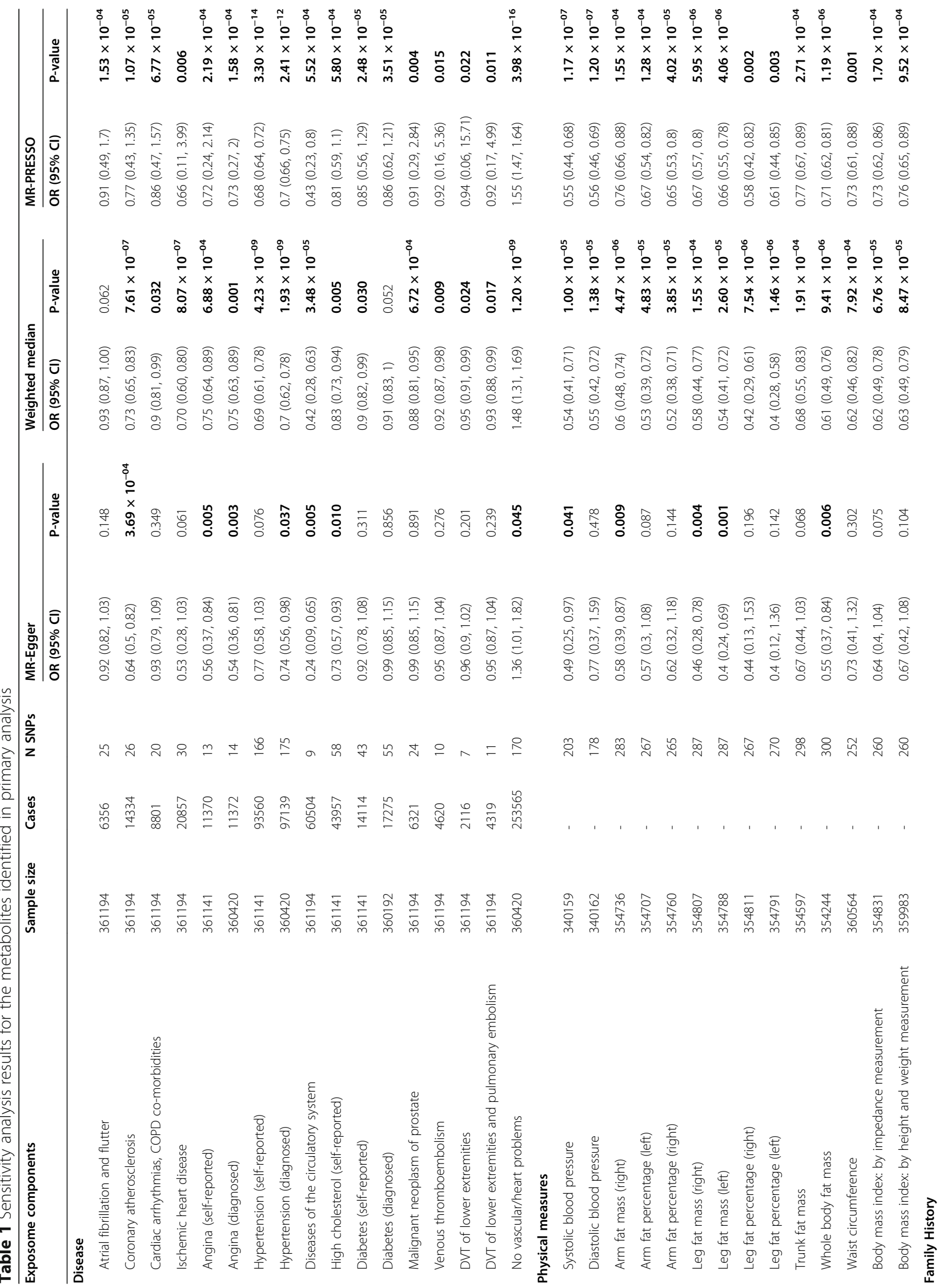




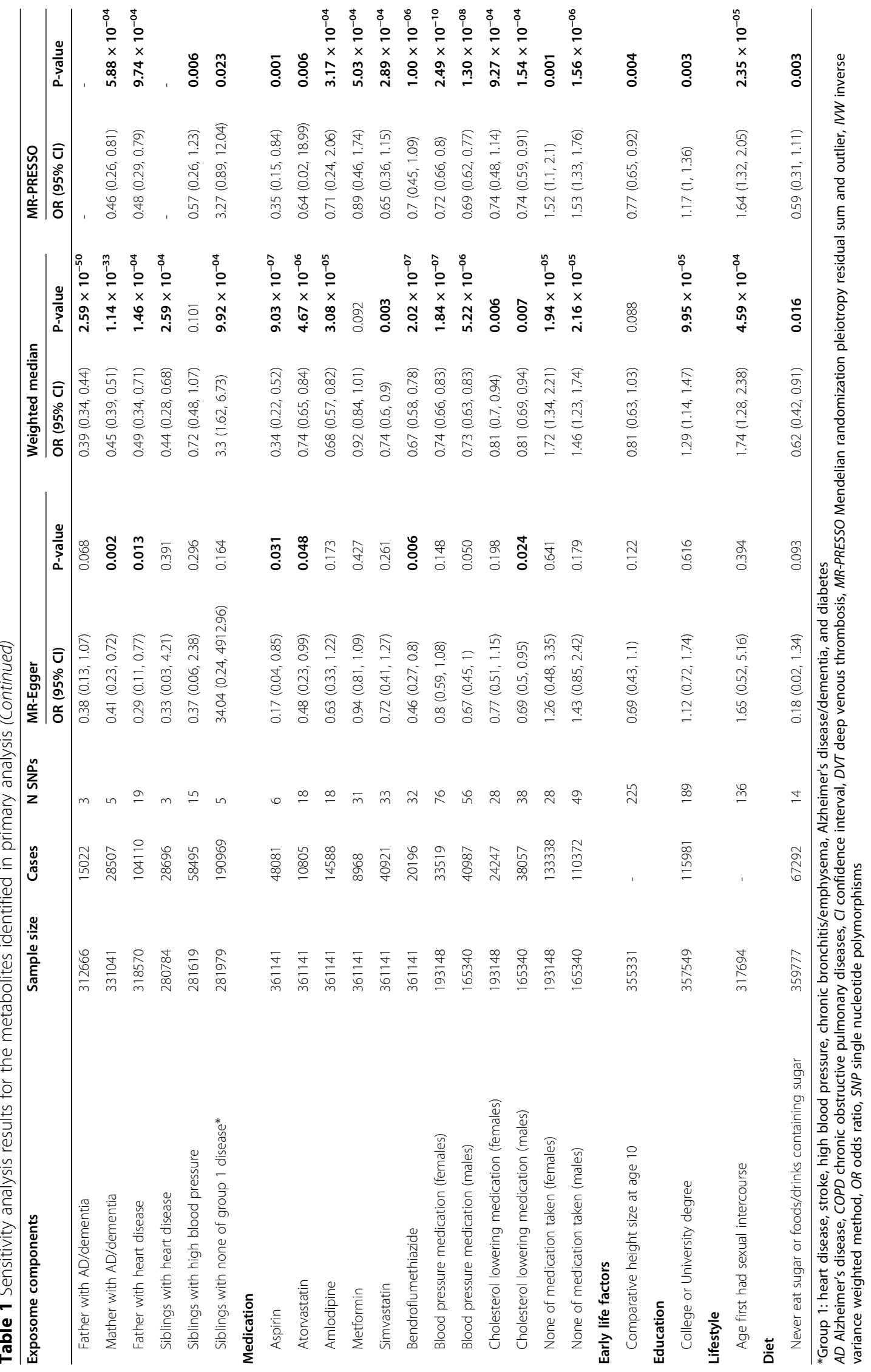




\begin{tabular}{|c|c|c|c|c|c|}
\hline Exposure & N SNPs & & & OR(95\% Cl) & FDR P-90 \\
\hline \multicolumn{6}{|l|}{ Disease } \\
\hline Atrial fibrillation and flutter & 25 & - & & $0.91(0.87,0.96)$ & 0.004 \\
\hline Coronary atherosclerosis & 26 & $\rightarrow$ & & $0.77(0.70,0.84)$ & $<0.001$ \\
\hline Cardiac arrhytmias, COPD co-morbidities & 20 & - & & $0.86(0.81,0.92)$ & $<0.001$ \\
\hline Ischaemic heart disease & 30 & $\longrightarrow$ & & $0.66(0.51,0.87)$ & 0.039 \\
\hline Angina (self-reported) & 13 & $\rightarrow$ & & $0.72(0.64,0.82)$ & $<0.001$ \\
\hline Angina (diagnosed) & 14 & $\rightarrow$ & & $0.73(0.65,0.83)$ & $<0.001$ \\
\hline Hypertension (self-reported) & 166 & - & & $0.68(0.62,0.74)$ & $<0.001$ \\
\hline Hypertension (diagnosed) & 175 & - & & $0.70(0.64,0.77)$ & $<0.001$ \\
\hline Diseases of the circulatory system & 9 & $\longrightarrow$ & & $0.43(0.32,0.59)$ & $<0.001$ \\
\hline High cholesterol (self-reported) & 58 & - & & $0.81(0.72,0.91)$ & 0.005 \\
\hline Diabetes (self-reported) & 43 & - & & $0.85(0.80,0.91)$ & $<0.001$ \\
\hline Diabetes (diagnosed) & 55 & - & & $0.86(0.81,0.92)$ & $<0.001$ \\
\hline Malignant neoplasm of prostate & 24 & - & & $0.91(0.86,0.97)$ & 0.023 \\
\hline Venous thromboembolism & 10 & * & & $0.92(0.87,0.97)$ & 0.039 \\
\hline DVT of lower extremities & 7 & - & & $0.94(0.91,0.98)$ & 0.043 \\
\hline DVT of lower extremities and pulmonary embolism & 11 & $=$ & & $0.92(0.88,0.97)$ & 0.026 \\
\hline No vascular/heart problems & 170 & & $\rightarrow$ & $1.55(1.41,1.71)$ & $<0.001$ \\
\hline \multicolumn{6}{|l|}{ Physical measures } \\
\hline Systolic blood pressure & 203 & $\rightarrow$ & & $0.55(0.44,0.68)$ & $<0.001$ \\
\hline Diastolic blood pressure & 178 & $\rightarrow$ & & $0.56(0.46,0.69)$ & $<0.001$ \\
\hline Arm fat mass (right) & 283 & $\rightarrow$ & & $0.76(0.66,0.88)$ & 0.003 \\
\hline Arm fat percentage (left) & 267 & $\rightarrow$ & & $0.67(0.54,0.82)$ & 0.002 \\
\hline Arm fat percentage (right) & 265 & 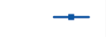 & & $0.65(0.53,0.80)$ & $<0.001$ \\
\hline Leg fat mass (right) & 287 & $\rightarrow$ & & $0.67(0.57,0.80)$ & $<0.001$ \\
\hline Leg fat mass (left) & 287 & $\rightarrow$ & & $0.66(0.55,0.78)$ & $<0.001$ \\
\hline Leg fat percentage (right) & 267 & $\longrightarrow$ & & $0.58(0.42,0.82)$ & 0.025 \\
\hline Leg fat percentage (left) & 270 & $\longrightarrow$ & & $0.61(0.44,0.85)$ & 0.042 \\
\hline Trunk fat mass & 298 & $\rightarrow$ & & $0.77(0.67,0.89)$ & 0.005 \\
\hline Whole body fat mass & 300 & $\rightarrow$ & & $0.71(0.62,0.81)$ & $<0.001$ \\
\hline Waist circumference & 252 & $\rightarrow$ & & $0.73(0.61,0.88)$ & 0.02 \\
\hline Body mass index: by impedance measurement & 260 & $\rightarrow$ & & $0.73(0.62,0.86)$ & 0.003 \\
\hline Body mass index: by height and weight measurement & 260 & $\rightarrow$ & & $0.76(0.65,0.89)$ & 0.014 \\
\hline \multicolumn{6}{|l|}{ Family History } \\
\hline Father with $\mathrm{AD} /$ dementia & 3 & $=$ & & $0.38(0.34,0.43)$ & $<0.001$ \\
\hline Mather with $\mathrm{AD} /$ dementia & 5 & $\rightarrow$ & & $0.46(0.39,0.54)$ & $<0.001$ \\
\hline Father with heart disease & 19 & $\longrightarrow-$ & & $0.48(0.33,0.69)$ & 0.002 \\
\hline Siblings with heart disease & 3 & $\rightarrow$ & & $0.39(0.26,0.60)$ & $<0.001$ \\
\hline Siblings with high blood pressure & 15 & $\longrightarrow$ & & $0.57(0.40,0.81)$ & 0.022 \\
\hline Siblings with none of group 1 disease ${ }^{*}$ & 5 & & & $3.27(1.71,6.24)$ & 0.006 \\
\hline \multicolumn{6}{|l|}{ Medication } \\
\hline Aspirin & 6 & - & & $0.35(0.25,0.49)$ & $<0.001$ \\
\hline Atorvastatin & 18 & $\longrightarrow$ & & $0.64(0.49,0.85)$ & 0.023 \\
\hline Amlodipine & 18 & $\rightarrow$ & & $0.71(0.61,0.82)$ & $<0.001$ \\
\hline Metformin & 31 & 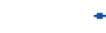 & & $0.89(0.84,0.95)$ & 0.006 \\
\hline Simvastatin & 33 & $\rightarrow$ & & $0.65(0.53,0.80)$ & 0.001 \\
\hline Bendroflumethiazide & 32 & $\rightarrow$ & & $0.70(0.62,0.79)$ & $<0.001$ \\
\hline Blood pressure medication (Females) & 76 & - & & $0.72(0.66,0.79)$ & $<0.001$ \\
\hline Blood pressure medication (Males) & 56 & $\rightarrow$ & & $0.69(0.62,0.77)$ & $<0.001$ \\
\hline Cholesterol lowering medication (Females) & 28 & $\rightarrow$ & & $0.74(0.64,0.87)$ & 0.004 \\
\hline Cholesterol lowering medication (Males) & 38 & $\Rightarrow$ & & $0.74(0.64,0.85)$ & $<0.001$ \\
\hline None of medication taken (Females) & 28 & & $\rightarrow$ & $1.52(1.21,1.90)$ & 0.005 \\
\hline None of medication taken (Males) & 49 & & $\rightarrow$ & $1.53(1.31,1.78)$ & $<0.001$ \\
\hline \multicolumn{6}{|l|}{ Early life factors } \\
\hline Comparative height size at age 10 & 225 & $\rightarrow$ & & $0.77(0.65,0.92)$ & 0.045 \\
\hline \multicolumn{6}{|l|}{ Education } \\
\hline College or University degree & 189 & & $\Rightarrow$ & $1.17(1.06,1.29)$ & 0.033 \\
\hline \multicolumn{6}{|l|}{ Lifestyle } \\
\hline Age first had sexual intercourse & 136 & & $\rightarrow$ & $1.64(1.32,2.05)$ & $<0.001$ \\
\hline \multicolumn{6}{|l|}{ Diet } \\
\hline Never eat sugar or foods/drinks containing sugar & 14 & $\longrightarrow-$ & & $0.59(0.44,0.80)$ & 0.009 \\
\hline & & $\begin{array}{l}0.50 \\
\text { ercentile }\end{array}$ & & & \\
\hline
\end{tabular}

Fig. 2 (See legend on next page.) 
(See figure on previous page.)

Fig. 2 Mendelian randomization estimates for association between genetically predicted exposures and longevity in primary analysis. The estimates present here were calculated by the IVW method. *Group 1: heart disease, stroke, high blood pressure, chronic bronchitis/emphysema, and Alzheimer's disease/dementia, diabetes. AD, Alzheimer's disease; Cl, confidence interval; COPD, chronic obstructive pulmonary diseases; DVT, deep venous thrombosis; FDR, false discovery rates; N, number or sample size; OR, odds ratio; SNPs, single nucleotide polymorphisms

were also associated with higher odds of 90th and 99th percentile longevity. After removing potential outlying SNP through MR-PRESSO outlier test, significant effects remained of the two traits (Table 2).

We also noted an association between self-reported high cholesterol $\left(\mathrm{OR}_{90}=0.81[0.72,0.91], \mathrm{P}_{90}=0.0003\right)$ and two longevity outcomes. After removing the outlying SNP identified by MR-PRESSO outlier test, the significant effects on 90th percentile longevity remained (Table 2). Besides, self-reported $\left(\mathrm{OR}_{90}=0.85[0.80\right.$, $\left.0.91], \mathrm{P}_{90}=2.2 \times 10^{-6}\right)$ and diagnosed $\left(\mathrm{OR}_{90}=0.86\right.$ $[0.81,0.92], \mathrm{P}_{90}=6.4 \times 10^{-6}$ ) diabetes showed robust causal effects on both 90th and 99th percentile longevity without evidence of heterogeneity or pleiotropy. Malignant neoplasm of prostate $\left(\mathrm{OR}_{90}=0.91[0.86,0.97], \mathrm{P}_{90}\right.$ $=0.0016)$ was also associated with lower odds of both two longevity outcomes without any evidence of heterogeneity or pleiotropy.

In the physical measures category, seven exposures referring to body morphology showed hazardous effects on both two longevity outcomes, including arm fat mass (right; $\mathrm{OR}_{90}=0.76[0.66,0.88], \mathrm{P}_{90}=0.0001$ ), arm fat percentage (right; $\mathrm{OR}_{90}=0.65[0.53,0.80], \mathrm{P}_{90}=3.0 \times$ $10^{-5}$ ), leg fat mass (right; $\mathrm{OR}_{90}=0.67[0.57,0.80], \mathrm{P}_{90}=$ $3.9 \times 10^{-6}$ ), leg fat mass (left; $\mathrm{OR}_{90}=0.66[0.55,0.78]$, $\left.\mathrm{P}_{90}=2.6 \times 10^{-6}\right)$, whole body fat mass $\left(\mathrm{OR}_{90}=0.71\right.$

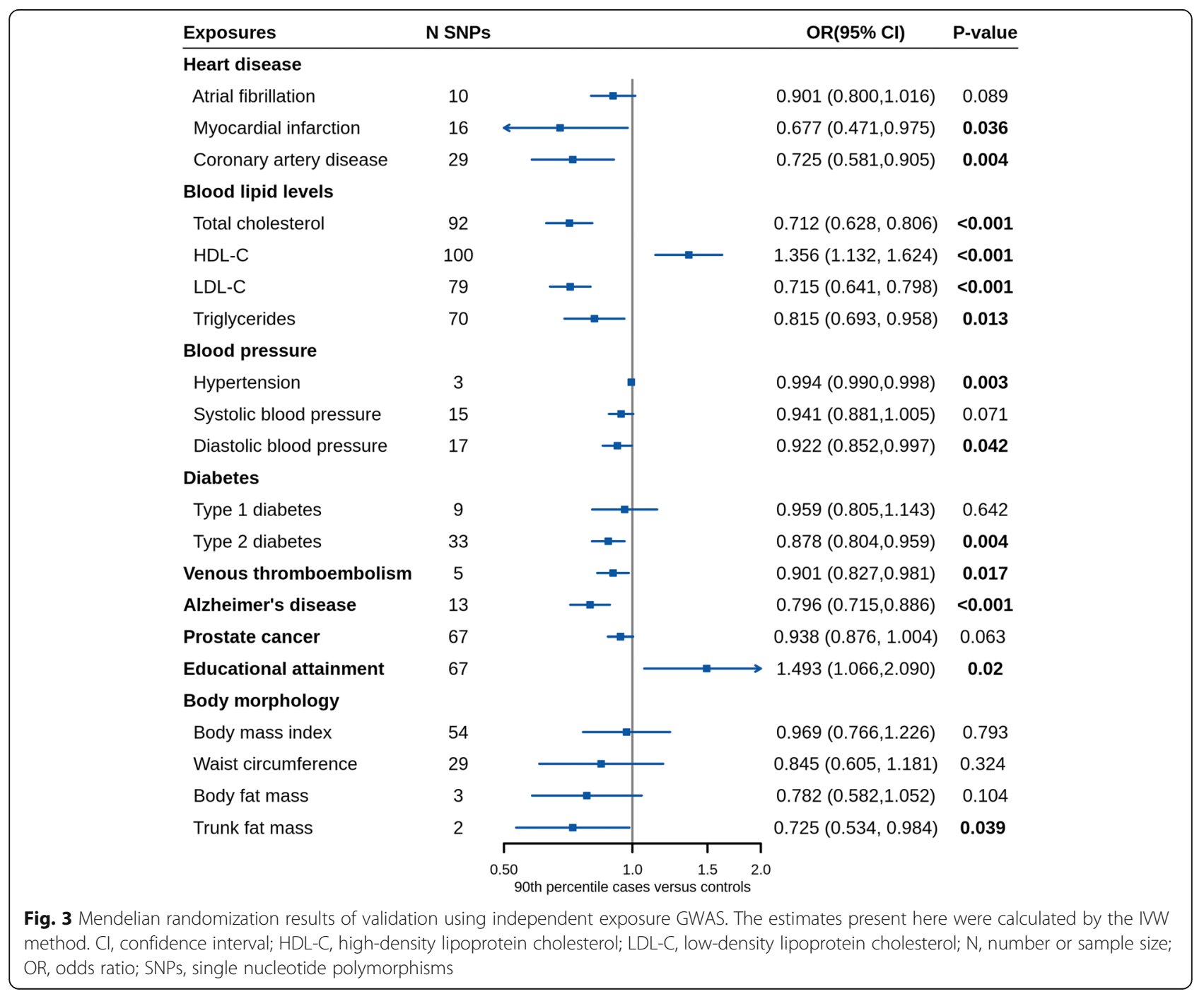


Table 2 Pleiotropy and heterogeneity analyses for the association between exposures and Alzheimer's disease in primary analysis

\begin{tabular}{|c|c|c|c|c|c|}
\hline \multirow[t]{2}{*}{ Exposome components } & \multirow{2}{*}{$\begin{array}{l}\text { MR-Egger } \\
\text { intercept } \\
\text { P-value }\end{array}$} & \multirow{2}{*}{$\begin{array}{l}\mathrm{Q} \text { test } \\
\mathrm{P} \text {-value }\end{array}$} & \multirow{2}{*}{$\begin{array}{l}\text { MR-PRES } \\
\text { SO global } \\
\text { test P-value }\end{array}$} & \multicolumn{2}{|c|}{ Corrected MR-PRESSO } \\
\hline & & & & OR $(95 \% \mathrm{Cl})$ & P-value \\
\hline \multicolumn{6}{|l|}{ Disease } \\
\hline Atrial fibrillation and flutter & 0.87 & 0.82 & 0.89 & - & - \\
\hline Coronary atherosclerosis & 0.11 & 0.08 & 0.16 & - & - \\
\hline Cardiac arrhythmias, COPD co-morbidities & 0.81 & 0.33 & 0.80 & - & - \\
\hline Ischemic heart disease & $1.66 \times 10^{-37}$ & 0.46 & $<0.001$ & $0.74(0.47,1.15)$ & $7.70 \times 10^{-06}$ \\
\hline Angina (self-reported) & 0.46 & 0.12 & 0.52 & - & - \\
\hline Angina (diagnosed) & 0.54 & 0.07 & 0.55 & - & - \\
\hline Hypertension (self-reported) & $1.11 \times 10^{-04}$ & 0.32 & $<0.001$ & - & - \\
\hline Hypertension (diagnosed) & $5.55 \times 10^{-05}$ & 0.73 & $<0.001$ & - & - \\
\hline Diseases of the circulatory system & 0.49 & 0.11 & 0.56 & - & - \\
\hline High cholesterol (self-reported) & $1.92 \times 10^{-07}$ & 0.32 & $<0.001$ & $0.79(0.62,1.01)$ & $5.16 \times 10^{-05}$ \\
\hline Diabetes (self-reported) & 0.28 & 0.36 & 0.29 & - & - \\
\hline Diabetes (diagnosed) & 0.24 & 0.07 & 0.25 & - & - \\
\hline Malignant neoplasm of prostate & 0.35 & 0.29 & 0.35 & - & - \\
\hline Venous thromboembolism & 0.19 & 0.35 & 0.31 & - & - \\
\hline DVT of lower extremities & 0.48 & 0.34 & 0.53 & - & - \\
\hline DVT of lower extremities and pulmonary embolism & 0.26 & 0.38 & 0.35 & - & - \\
\hline No vascular/heart problems & $2.01 \times 10^{-04}$ & 0.34 & 0.001 & $1.53(1.45,1.62)$ & $1.43 \times 10^{-15}$ \\
\hline \multicolumn{6}{|l|}{ Physical measures } \\
\hline Systolic blood pressure & $2.16 \times 10^{-08}$ & 0.72 & $<0.001$ & $0.54(0.44,0.66)$ & $3.10 \times 10^{-08}$ \\
\hline Diastolic blood pressure & $1.06 \times 10^{-04}$ & 0.39 & $<0.001$ & $0.55(0.45,0.67)$ & $1.39 \times 10^{-08}$ \\
\hline Arm fat mass (right) & 0.09 & 0.17 & 0.09 & - & - \\
\hline Arm fat percentage (left) & 0.002 & 0.61 & 0.002 & $0.65(0.53,0.8)$ & $4.99 \times 10^{-05}$ \\
\hline Arm fat percentage (right) & 0.005 & 0.87 & 0.005 & $0.64(0.52,0.78)$ & $1.42 \times 10^{-05}$ \\
\hline Leg fat mass (right) & 0.11 & 0.14 & 0.13 & - & - \\
\hline Leg fat mass (left) & 0.03 & 0.06 & 0.03 & $0.67(0.57,0.8)$ & $8.71 \times 10^{-06}$ \\
\hline Leg fat percentage (right) & $2.94 \times 10^{-28}$ & 0.64 & $<0.001$ & $0.51(0.4,0.65)$ & $8.76 \times 10^{-08}$ \\
\hline Leg fat percentage (left) & $4.36 \times 10^{-23}$ & 0.48 & $<0.001$ & $0.53(0.43,0.67)$ & $1.15 \times 10^{-07}$ \\
\hline Trunk fat mass & 0.01 & 0.51 & 0.02 & $0.78(0.68,0.9)$ & $4.58 \times 10^{-04}$ \\
\hline Whole body fat mass & 0.10 & 0.22 & 0.11 & - & - \\
\hline Waist circumference & $2.90 \times 10^{-05}$ & 0.99 & $<0.001$ & $0.7(0.58,0.84)$ & $1.83 \times 10^{-04}$ \\
\hline Body mass index: by impedance measurement & $3.11 \times 10^{-09}$ & 0.60 & $<0.001$ & $0.69(0.6,0.79)$ & $8.48 \times 10^{-08}$ \\
\hline Body mass index: by height and weight measurement & $6.25 \times 10^{-08}$ & 0.60 & $<0.001$ & $0.72(0.63,0.82)$ & $1.06 \times 10^{-06}$ \\
\hline \multicolumn{6}{|l|}{ Family history } \\
\hline Father with $\mathrm{AD} /$ dementia & 0.41 & 0.89 & - & - & - \\
\hline Mather with AD/dementia & 0.18 & 0.15 & 0.40 & - & - \\
\hline Father with heart disease & 0.006 & 0.24 & 0.01 & $0.5(0.35,0.72)$ & $2.47 \times 10^{-04}$ \\
\hline Siblings with heart disease & 0.25 & 0.85 & - & - & - \\
\hline Siblings with high blood pressure & 0.02 & 0.64 & 0.02 & $0.65(0.3,1.41)$ & 0.016 \\
\hline Siblings with none of group 1 disease* & 0.18 & 0.31 & 0.30 & - & - \\
\hline \multicolumn{6}{|l|}{ Medication } \\
\hline Aspirin & 0.46 & 0.24 & 0.59 & - & - \\
\hline Atorvastatin & $2.15 \times 10^{-33}$ & 0.36 & $<0.001$ & $0.72(0.41,1.26)$ & $8.30 \times 10^{-07}$ \\
\hline
\end{tabular}


Table 2 Pleiotropy and heterogeneity analyses for the association between exposures and Alzheimer's disease in primary analysis (Continued)

\begin{tabular}{|c|c|c|c|c|c|}
\hline \multirow[t]{2}{*}{ Exposome components } & \multirow{2}{*}{$\begin{array}{l}\text { MR-Egger } \\
\text { intercept } \\
\text { P-value }\end{array}$} & \multirow{2}{*}{$\begin{array}{l}\mathrm{Q} \text { test } \\
\mathrm{P} \text {-value }\end{array}$} & \multirow{2}{*}{$\begin{array}{l}\text { MR-PRES } \\
\text { SO global } \\
\text { test P-value }\end{array}$} & \multicolumn{2}{|c|}{ Corrected MR-PRESSO } \\
\hline & & & & OR $(95 \% \mathrm{Cl})$ & P-value \\
\hline Amlodipine & 0.04 & 0.72 & 0.05 & - & - \\
\hline Metformin & 0.70 & 0.48 & 0.70 & - & - \\
\hline Simvastatin & $3.75 \times 10^{-07}$ & 0.67 & $<0.001$ & $0.63(0.42,0.93)$ & $1.19 \times 10^{-05}$ \\
\hline Bendroflumethiazide & 0.18 & 0.11 & 0.21 & - & - \\
\hline Blood pressure medication (females) & 0.02 & 0.50 & 0.03 & - & - \\
\hline Blood pressure medication (males) & 0.04 & 0.86 & 0.04 & - & - \\
\hline Cholesterol lowering medication (females) & $1.55 \times 10^{-06}$ & 0.87 & $<0.001$ & $0.71(0.56,0.91)$ & $9.32 \times 10^{-06}$ \\
\hline Cholesterol lowering medication (males) & $1.70 \times 10^{-05}$ & 0.66 & $<0.001$ & $0.71(0.62,0.82)$ & $3.07 \times 10^{-06}$ \\
\hline None of medication taken (females) & 0.010 & 0.70 & 0.01 & $1.62(1.31,2)$ & $3.05 \times 10^{-05}$ \\
\hline None of medication taken (males) & 0.002 & 0.80 & 0.002 & $1.61(1.43,1.81)$ & $4.23 \times 10^{-08}$ \\
\hline \multicolumn{6}{|l|}{ Early life factors } \\
\hline Comparative height size at age 10 & 0.004 & 0.62 & 0.006 & $0.78(0.66,0.93)$ & 0.006 \\
\hline \multicolumn{6}{|l|}{ Education } \\
\hline College or university degree & $2.17 \times 10^{-04}$ & 0.84 & $<0.001$ & $1.2(1.07,1.35)$ & $2.03 \times 10^{-04}$ \\
\hline \multicolumn{6}{|l|}{ Lifestyle } \\
\hline Age first had sexual intercourse & 0.18 & 1.00 & 0.19 & - & - \\
\hline \multicolumn{6}{|l|}{ Diet } \\
\hline Never eat sugar or foods/drinks containing sugar & 0.54 & 0.22 & 0.58 & - & - \\
\hline
\end{tabular}

*Group 1: heart disease, stroke, high blood pressure, chronic bronchitis/emphysema, Alzheimer's disease/dementia, and diabetes $A D$ Alzheimer's disease, COPD chronic obstructive pulmonary diseases, Cl confidence interval, DVT deep venous thrombosis, MR-PRESSO Mendelian randomization pleiotropy residual sum and outlier, IVW inverse variance weighted method, OR odds ratio

$\left.[0.62,0.81], \mathrm{P}_{90}=7.1 \times 10^{-7}\right)$, waist circumference $\left(\mathrm{OR}_{90}\right.$ $\left.=0.73[0.61,0.88], \mathrm{P}_{90}=0.0012\right)$, and body mass index (BMI) measured by impedance measurement $\left(\mathrm{OR}_{90}=\right.$ $\left.0.73[0.62,0.86], \mathrm{P}_{90}=0.0001\right)$. Results of arm fat mass (right), leg fat mass (right), and whole body fat mass were robust for that no evidence of directional pleiotropy or heterogeneity were identified. Results of arm fat percentage (right), waist circumference, and BMI measured by impedance measurement showed potential pleiotropy, but they remain significant after removing outliers (Table 2). Remarkably, sitting height, standing height, weight, and body fat-free mass were unrelated with human longevity in a high power (see Additional file 1: Table S10 for more unassociated results).

Exposures of medication or family history were correlated with significant exposures of the disease category, including Alzheimer's disease (AD) or dementia (father with $\mathrm{AD} /$ dementia: $\mathrm{OR}_{90}=0.38[0.34,0.43], \mathrm{P}_{90}=6.0 \times$ $10^{-61}$; mother with $\mathrm{AD} /$ dementia: $\mathrm{OR}_{90}=0.46[0.39$, 0.54], $\mathrm{P}_{90}=4.9 \times 10^{-23}$ ), heart disease (father with heart disease: $\mathrm{OR}_{90}=0.48[0.33,0.69], \mathrm{P}_{90}=8.4 \times 10^{-5}$; siblings with heart disease: $\mathrm{OR}_{90}=0.39[0.26,0.60], \mathrm{P}_{90}=$ $1.7 \times 10^{-5}$ ), hypertension (siblings with high blood pressure: $\left.\mathrm{OR}_{90}=0.57[0.40,0.81], \mathrm{P}_{90}=0.0014\right)$, and diabetes, chronic bronchitis/emphysema, stroke, and high cholesterol (siblings with none of these diseases: $\mathrm{OR}_{90}=$ $\left.3.27[1.71,6.24], \mathrm{P}_{90}=0.0003\right)$. Regular taken of blood pressure medication, cholesterol lowering medication, metformin, and aspirin also showed significant association with lower odds of 90th and 99th percentile longevity. Besides, more medications taken $\left(\mathrm{OR}_{90}=0.37\right.$ $\left.[0.18,0.77], \mathrm{P}_{90}=0.008\right)$ was suggestively associated with lower odds of surviving to the 90th and 99th percentile age (see Additional file 1: Table S11-S12), while none medications taken showed the opposite (Fig, 2). In medication and family history category, results either showed no potential pleiotropy in MR-Egger intercept test or remained significant on 90th percentile longevity after removing outlying SNPs (Table 2).

Additionally, we found that comparative height size at age $10\left(\mathrm{OR}_{90}=0.77[0.65,0.92], \mathrm{P}_{90}=0.0035\right)$ and never eat sugar or food/drinks containing sugar $\left(\mathrm{OR}_{90}=0.59\right.$ $\left.[0.44,0.80], \mathrm{P}_{90}=0.0005\right)$ showed association with lower odds of 90th and 99th percentile longevity. Substantial pleiotropy was only detected in comparative height size at age 10 (MR-Egger intercept $\mathrm{P}=0.004$; global test $\mathrm{P}=$ 0.006), but the result of corrected MR-PRESSO test was still significant after removing outlying variants.

Comparing results of 53 reported exposures in primary and in secondary analysis, four traits in disease category 
were only associated with 90th percentile survival longevity, including atrial fibrillation and flutter $\left(\mathrm{OR}_{90}=\right.$ $\left.0.91[0.87,0.96], \mathrm{P}_{90}=0.0002\right)$, deep venous thrombosis $(\mathrm{DVT})$ of lower extremities $\left(\mathrm{OR}_{90}=0.94[0.91,0.98], \mathrm{P}_{90}\right.$ $=0.0033)$, DVT of lower extremities and pulmonary embolism $\left(\mathrm{OR}_{90}=0.92[0.88,0.97], \mathrm{P}_{90}=0.0018\right)$, and venous thromboembolism $\left(\mathrm{VTE} ; \mathrm{OR}_{90}=0.92[0.87,0.97]\right.$, $\left.\mathrm{P}_{90}=0.0028\right)$. Five body morphology traits in physical measures category were associated with lower odds of 90th percentile longevity, including arm fat percentage (left; $\mathrm{OR}_{90}=0.67[0.54,0.82], \mathrm{P}_{90}=0.0001$ ), leg fat percentage (right; $\mathrm{OR}_{90}=0.58[0.42,0.82], \mathrm{P}_{90}=0.0017$ ), leg fat percentage (left; $\mathrm{OR}_{90}=0.61[0.44,0.85], \mathrm{P}_{90}=$ 0.0032), trunk fat mass $\left(\mathrm{OR}_{90}=0.77[0.67,0.89], \mathrm{P}_{90}=\right.$ 0.0002 ), and BMI measured by height and weight measurement $\left(\mathrm{OR}_{90}=0.76[0.65,0.89], \mathrm{P}_{90}=0.0008\right)$. Besides, college or university degree $\left(\mathrm{OR}_{90}=1.17[1.06\right.$, 1.29], $\left.\mathrm{P}_{90}=0.0024\right)$ and age first had sexual intercourse $\left(\mathrm{OR}_{90}=1.64[1.32,2.05], \mathrm{P}_{90}=1.2 \times 10^{-5}\right)$ were associated with higher odds of longevity only in 90th percentile data. All significant exposures identified in secondary analysis also showed significant results in primary stage.

\section{Validation}

In the validation, the results of myocardial infarction $\left(P_{90}=0.036\right)$, coronary artery disease $\left(P_{90}=0.004\right)$, VTE $\left(\mathrm{P}_{90}=0.017\right), \mathrm{AD}\left(\mathrm{P}_{90}=3.0 \times 10^{-5}\right)$, trunk fat mass $\left(\mathrm{P}_{90}\right.$ $=0.039$ ), and education attainment (i.e., the number of years of schooling completed; $\mathrm{P}_{90}=0.020$ ) had secured our MR estimates in screening. Causal effects of LDL-C $\left(\mathrm{OR}_{90}=0.72[0.64,0.80], \mathrm{P}_{90}=2.3 \times 10^{-9}\right)$, total cholesterol $\left(\mathrm{OR}_{90}=0.71[0.63,0.81], \mathrm{P}_{90}=9.4 \times 10^{-8}\right)$, HDL-C $\left(\mathrm{OR}_{90}=1.36[1.13,1.62], \mathrm{P}_{90}=0.001\right)$, triglycerides $\left(\mathrm{OR}_{90}=0.82[0.70,0.96], \mathrm{P}_{90}=0.013\right)$, and type 2 diabetes $\left(\mathrm{T} 2 \mathrm{D} ; \mathrm{OR}_{90}=0.88[0.80,0.96], \mathrm{P}_{90}=0.004\right)$ were validated and supplemented our screening results of high cholesterol and diabetes. Atrial fibrillation $\left(\mathrm{OR}_{90}=\right.$ $\left.0.90[0.80,1.02], \mathrm{P}_{90}=0.089\right)$, prostate cancer $\left(\mathrm{OR}_{90}=\right.$ $\left.0.94[0.88,1.00], \mathrm{P}_{90}=0.063\right)$, type 1 diabetes $\left(\mathrm{OR}_{90}=\right.$ $\left.0.96[0.81,1.14], \mathrm{P}_{90}=0.642\right)$, $\mathrm{BMI}\left(\mathrm{OR}_{90}=0.97[0.77\right.$, 1.23], $\left.\mathrm{P}_{90}=0.79\right)$, and waist circumference $\left(\mathrm{OR}_{90}=0.85\right.$ $\left.[0.61,1.18], \mathrm{P}_{90}=0.324\right)$ were well-powered but showed no causal effects on longevity (see Additional file 1: Table S10-S11). SBP and body fat mass were nonsignificant in the validation, but the statistical power to detect an effect was not enough to preclude the positive effects in primary analysis. Of all exposures in the validation, the Egger intercept test showed no pleiotropy.

After adjusting for genetic correlation between different types of blood lipids, the association between HDL$\mathrm{C}$ and longevity was partially attenuated $\left(\mathrm{OR}_{90}=1.29\right.$ $\left.[1.05,1.60], \mathrm{P}_{90}=0.016\right)$. The hazardous effect of triglycerides was fully disappeared $\left(\mathrm{OR}_{90}=0.99[0.81,1.21]\right.$,
$\left.\mathrm{P}_{90}=0.927\right)$, while that of LDL-C was still significant $\left(\mathrm{OR}_{90}=0.68[0.62,0.76], \mathrm{P}_{90}=3.6 \times 10^{-13}\right)$.

\section{Potential components of human longevity exposome}

After screening and validation, robust exposures were considered components of longevity exposome, including 39 exposures that showed associations with both 90th and 99th percentile survival longevity at significant or suggestive levels in screening (see Additional file 1: Table S3), as well as VTE, AD, trunk fat mass, and educational attainment that were significant in the validation (Figs. 3 and 4). For note, malignant neoplasm of prostate, BMI, and waist circumference were excluded because of the non-significant validation results with a high power. Atrial fibrillation and flutter and age first had sexual intercourse were not considered components of longevity exposome for that the two results cannot be verified neither in secondary analysis nor in validation.

\section{Discussion}

This is the first study using the MR approach to reveal causal components of longevity exposome. We found evidence that some heart diseases, metabolic syndromes, $\mathrm{AD}, \mathrm{VTE}$, greater body fat, higher comparative height size at 10, and never eat sugar or foods/drinks containing sugar have adverse effects on longevity, whereas higher HDL-C levels and higher education attainment have protective effects.

Our findings suggest the susceptibility to age-related diseases may significantly affect human longevity. Intuitively, our results have shown consistency with previous investigations. A progressive delay in the onset of agerelated diseases, including ischemic heart disease, coronary atherosclerosis, angina, and $\mathrm{AD}$, has been found with an association of increasing survival age [22]. Remarkably, GWAS have found that human longevity shared genetic correlations with CVD [3]. However, previous studies didn't investigate the potential association using robust genetic analyses. By using MR method, we strengthen the potential causal effects of cardiovascular diseases on human longevity. Our MR study also demonstrated that hypertension, T2D, and higher LDL-C level were associated with lower odds of longevity, which is a strong confirmation of previous observational studies $[2,5,8,23]$. It is believed to be causing genomic instability, telomere attrition, epigenetic alterations, and loss of proteostasis in the development of metabolic syndrome [24], thus leading to the reducing survival age. A healthy metabolic profile to avoid or delay the occurrence of metabolic syndrome may prolong longevity, as our results yield a positive association between age high blood pressure diagnosed and longevity at a suggestive level (see Additional file 1: Table S10-S12). Previous studies have also shown correlations between 


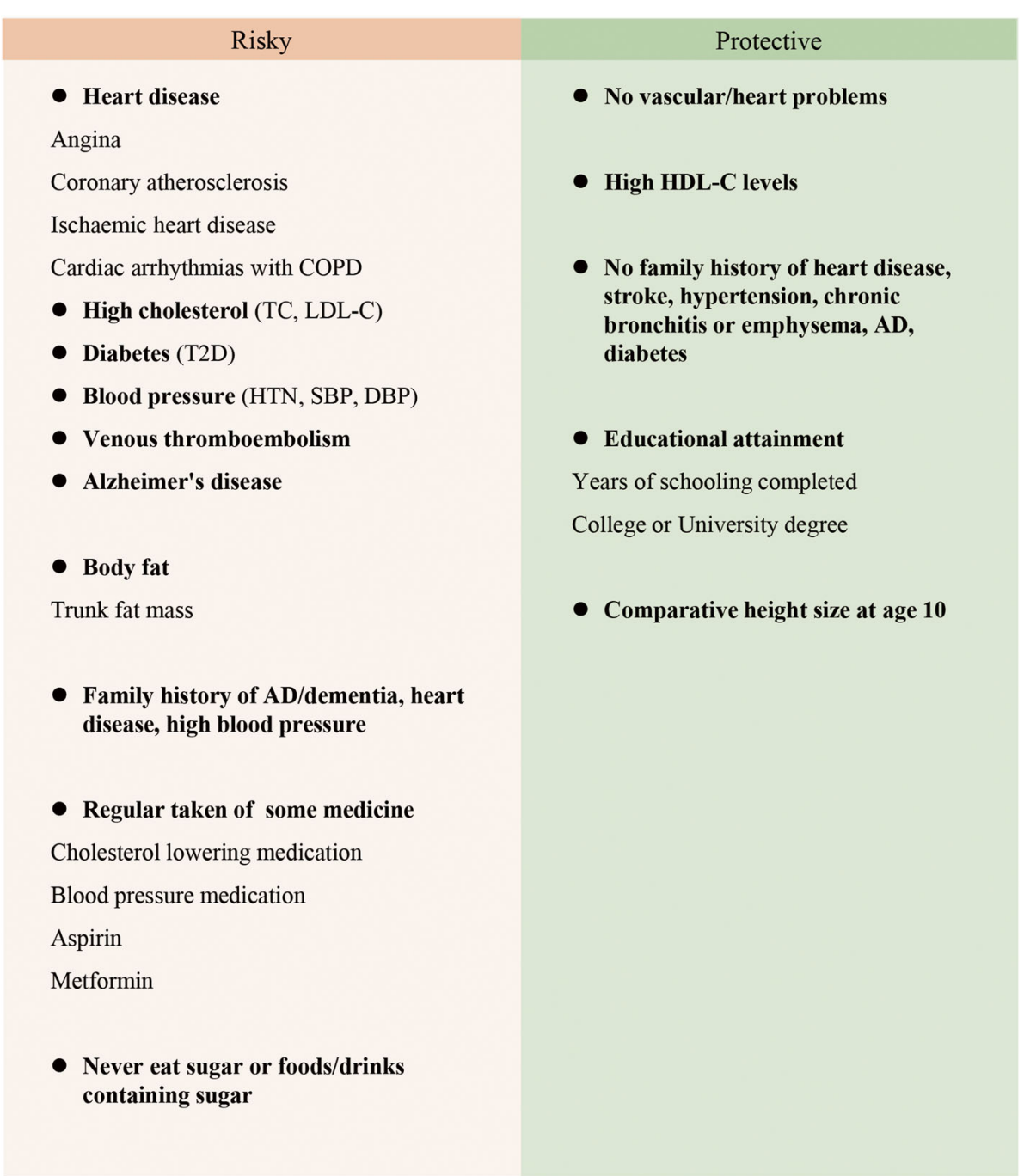

Fig. 4 Components of the longevity exposome. COPD, chronic obstructive pulmonary diseases; DBP, diastolic blood pressure; HTN, hypertension; HDL-C, high-density lipoprotein cholesterol; LDL-C, low-density lipoprotein cholesterol; SBP, systolic blood pressure; T2D, type 2 diabetes; TC, total cholesterol

exceptionally healthy metabolic profile and human longevity [5, 25], shedding new insights for revealing the complexity of longevity. Furthermore, it is well known that many of those metabolic factors act as risk factors for CVD, metabolic syndrome, and AD. As these exposures of longevity interplay and intertwined, further studies are needed to decipher the pathways supporting these causal associations.

The protective effect of HDL-C was still significant in our study even after adjusting for LDL-C and triglycerides. As the genetically predicted HDL-C is not causally associated with CVD [26, 27], the relationship between HDL-C and longevity is unexpected and the underlying mechanism is not clear. HDL-C levels may affect longevity through complex relationships involving diverse factors [28]. Future studies focusing on the quality and components of HDL rather than the simple measurement of HDL may help to clarify the underlying mechanisms behind this relationship.

Despite some published studies have indicated an association between BMI and human lifespan [2], our results for BMI were conflicted among screening and validation stage. The conflicting results may be attributed to non-linear relation between BMI and longevity. As previous MR study and observational study showed, the relation between BMI and all-cause mortality is $\mathrm{J}$ shaped [29,30], and underweight is also correlated with higher risk of mortality. On the other hand, the relation of BMI and mortality is also affected by smoking status and age [29]. Thus, it is reasonable not to simply include higher BMI into the hazardous components of longevity exposome. However, some traits of body fat showed 
robust association with longevity, while body fat-free mass and weight were unrelated to longevity (see Additional file 1: Table S10). Based on these results, in terms of longevity, a practical recommendation is to reduce body fat than focus on the body fat-free mass or weight. Especially, higher trunk fat mass showed an association with human longevity. As a marker of central adiposity, it was linked with an increased risk of CVD and metabolic diseases [31], which may be one of the potential mechanisms.

Height in adulthood is believed to link with health and longevity, but the exact effect of height on human longevity is conflicted [32-35]. Our study clarified that standing height and sitting height were not associated with longevity at a suggestive level (see Additional file 1: Table S10-S12). However, higher comparative height size at 10 was negatively associated with human longevity. This result provided a different research prospective for investigation of relation between height and longevity.

Our results indicated a protective effect of higher education attainment, especially gaining college or university degree, on longevity. It is supported by previous evidences that higher life expectancies are associated with greater educational levels [36-38]. Education has also been proposed as a protective factor with both AD and CVD outcomes [39, 40]. Whether the protective effect of education on longevity is achieved by reducing the risk of CVD or $\mathrm{AD}$ needs further investigations.

Strengths of the study include the adoption of the MR approach for assessing the causal effects of a wide array of factors, getting the utmost out of large data and reducing selection bias. Our study identified some exposures that have never been investigated with MR frameworks of longevity, such as VTE, family history, body fat, diet, and comparative height in early life. Furthermore, the prudency on the definition of longevity phenotype has also allowed us to propose components of exposome causally linked to longevity more precisely since the definition of outcome was limited to mortality or parental life span in previous MR $[2,8]$. Meanwhile, as with all MR studies, the exclusion of pleiotropy or alternative direct causal pathways is a conspicuous challenge. Although all the reported causal exposures in this study identified no pleiotropy in the Egger intercept test, significant Q-tests for some traits found substantial heterogeneity in the analysis. However, to avoid violation of MR assumptions, we conducted sensitivity analysis with weighted median, MR-Egger, and MR-PRESSO method. These methods can provide unbiased causal effect estimates at the cost of reduced power when invalid IVs exist [16, 17], and MR-PRESSO outlier test can return an unbiased result by removing potential outlying SNPs [18]. For each significant causal exposure in screening, the point estimates in sensitivity analyses were consistent with IVW, enhancing the robustness of our results [12]. Moreover, increased confidences were gained from the validation using independent exposure datasets. For exposures with vague phenotype descriptions in UKB, more detailed causal traits like LDL-C and T2D were included in the validation analysis using non-UKB exposure data.

There are some limitations to the present study. First, although we have used the largest data of longevity [3], the power of some exposures was below $80 \%$. For example, smoking-related traits showed non-significant effects on longevity; however, because of the limited power (Additional file 2: Table S13S14), we cannot preclude that they have effects on longevity. Second, not all significant exposures were able to conduct validation due to the lack of appropriate non-UKB data. It is important to note that the absence of a validation result does not disconfirm the robustness of a causal factor, but it also points to the need for further studies with a more comprehended exposure phenotype and a large sample size. Third, some of the exposures from UKB are ordinal variables but are treated as continuous when calculating betas for effect allele at each SNP, leading to difficulties in interpreting estimates quantitatively in subsequent MR analysis. In addition, the findings were discovered from participants of European ancestry that were recruited at the age between 40 and 69 that may not be generalizable to other populations [11]. Another limitation is that for some exposure GWASs, only sex stratified data were available in UK Biobank given that the outcome dataset is men and women combined. However, the effect estimates were very similar between men and women (Fig. 2), indicating the results were reliable. What is more, a few SNPs overlapped among some exposures, which may suggest that these exposures affect longevity by an interaction. Further studies are required to clarify the underpinning mechanisms of those causal associations.

Based on our findings, it is pellucid that the interventions on cardiovascular disease, metabolic syndrome, and AD, as well as VTE are in demand for the overall benefits of human longevity. Several preventions strategies have been proposed in published literatures and should be abundantly publicized [24, 39, 40]. We recommend people reducing body fat mass, especially the trunk fat mass, rather than simply focusing on losing weight. In the long term, receiving a higher-level education, at least gaining college or university degree, can generate persistent benefits for longevity. Moreover, appropriate intake of sugar or food/drinks containing sugar is recommended for the general population. 


\section{Conclusions}

In conclusion, by screening thousands of environmental factors for their association with human longevity in a MR framework, we proposed potential components of exposome that were causally linked to longevity. Our results supported the previous results that some agerelated diseases, such as heart diseases, metabolic syndromes, and $\mathrm{AD}$, are causally related to longevity. And we first reported the association between venous thromboembolism, never eat sugar or foods/drinks containing sugar, comparative height size at 10, and longevity. We also highlighted some powerful unrelated associations, such as sitting height, standing height, weight, and body fat-free mass. Prevention strategies should focus on modifying these risk factors and promote protective recommendations to improve longevity.

\section{Abbreviations}

AD: Alzheimer's disease; CVD: Cardiovascular disease; DBP: Diastolic blood pressure; FDR: False discovery rates; GWAS: Genome-wide association studies; HDL-C: High-density lipoprotein cholesterol; IV: Instrumental variables; IVW: Inverse variance weighted; LDL-C: Low-density lipoprotein cholesterol; MR: Mendelian randomization; MR-PRESSO: Mendelian randomization pleiotropy residual sum and outlier; OR: Odds ratios; SBP: Systolic blood pressure; SNP: Single nucleotide polymorphisms; T2D: Type 2 diabetes; VTE: Venous thromboembolism

\section{Supplementary Information}

The online version contains supplementary material available at https://doi. org/10.1186/s12916-021-02030-4.

Additional file 1: Table S1. Details of the instruments used as proxy risk factors for longevity. Table S2. Description of independent exposure GWAS used in validation. Table S3. Estimates of inverse variance weighted method for significant associations between genetically predicted exposures and longevity. Table S4. Sensitivity analysis results for the metabolites identified in secondary analysis. Table S5. Pleiotropy and heterogeneity analyses for the association between exposures and Alzheimer's disease in secondary analysis. Table S6. The Mendelian randomization association of individual SNPs of screening traits in primary analysis. Table S7. Mendelian randomization results of validation using independent exposure GWAS. Table S8. Mendelian randomization results of validation using dataset of 90th percentile longevity. Table S9. Mendelian randomization results of validation using dataset of 99th percentile longevity. Table S10. List of exposures in the present study. Table S11. Exposures identified significantly and suggestively associated with 90th percentile longevity in primary analyses. Table S12. Exposures identified significantly and suggestively associated with 99th percentile longevity in secondary analyses.

Additional file 2: Table S13. Mendelian randomization results of all traits in primary analysis using dataset of 90th percentile longevity. Table S14. Mendelian randomization results of all traits in secondary analysis using dataset of 99th percentile longevity.

\section{Acknowledgements}

This work was made possible by the generous sharing of GWAS summary statistics. We thank the Neale Lab for offering the GWAS of exposure data. We also thank the UK Biobank and the International Genomics of Alzheimer's Project (IGAP) for providing summary results data for these analyses. The investigators within IGAP contributed to the design and implementation of IGAP and/or provided data but did not participate in analysis or writing of this report. IGAP was made possible by the generous participation of the control subjects, the patients, and their families. The i-Select chips were funded by the French National Foundation on Alzheimer's disease and related disorders. EADI was supported by the LABEX (laboratory of excellence program investment for the future) DISTALZ grant, Inserm, Institut Pasteur de Lille, Université de Lille 2, and the Lille University Hospital. GERAD was supported by the Medical Research Council (grant no. 503480), Alzheimer's Research UK (grant no. 503176), the Wellcome Trust (grant no. 082604/2/07/Z), and German Federal Ministry of Education and Research (BMBF): Competence Network Dementia (CND) grant no. 01Gl0102, 01Gl0711, and 01GI0420. CHARGE was partly supported by the NIH/NIA grant R01 AG033193 and the NIA AG081220 and AGES contract N01-AG-12100, the NHLBI grant R01 HL105756, the Icelandic Heart Association, and the Erasmus Medical Center and Erasmus University. ADGC was supported by the NIH/NIA grants: U01 AG032984, U24 AG021886, U01 AG016976, and the Alzheimer's Association grant ADGC-10-196728.

\section{Authors' contributions}

JTY had full access to all of the data in the study and take responsibility for the integrity of the data and the accuracy of the data analysis.

Concept and design: JTY. Acquisition, analysis, or interpretation of data: all authors. Drafting of the manuscript: SYH, YXY, SDC, HQL, XQZ, CZ, KK, and LF. Critical revision of the manuscript for important intellectual content: all authors. Statistical analysis: SYH and YXY. Obtained funding: JTY. Administrative, technical, or material support: LT, JTY, and QD. The authors read and approved the final manuscript.

\section{Funding}

This study was supported by grants from the National Natural Science Foundation of China (91849126); the National Key R\&D Program of China (2018YFC1314700); Shanghai Municipal Science and Technology Major Project (No.2018SHZDZX01); ZJLab, Shanghai Center for Brain Science and Brain-Inspired Technology, Tianqiao; and Chrissy Chen Institute, and the State Key Laboratory of Neurobiology and Frontiers Center for Brain Science of Ministry of Education, Fudan University. The funding bodies had no role in the design of the study and collection, analysis, and interpretation of data, or in writing the manuscript.

\section{Availability of data and materials}

All the data used in this study can be acquired from the online data repository of Neale Lab, MR-Base, or from the individual referenced papers. Any other data generated in the analysis process can be requested from the corresponding author.

\section{Declarations}

\section{Ethics approval and consent to participate}

This study is based on publicly available summarized data. Individual studies within each genome-wide association study received approval from a relevant institutional review board, and informed consent was obtained from participants or from a caregiver, legal guardian, or other proxy.

\section{Consent for publication}

Not applicable.

\section{Competing interests}

The authors declare that they have no competing interests.

\section{Author details}

${ }^{1}$ Department of Neurology and Institute of Neurology, Huashan Hospital, State Key Laboratory of Medical Neurobiology and MOE Frontiers Center for Brain Science, Shanghai Medical College, Fudan University, 12th Wulumuqi Zhong Road, Shanghai 200040, China. ²Department of Neurology, Qingdao Municipal Hospital, Qingdao University, Qingdao, China. ${ }^{3}$ Department of Psychological Medicine, Yong Loo Lin School of Medicine, National University of Singapore, Singapore, Singapore. ${ }^{4}$ Genetics and Aging Research Unit, McCance Center for Brain Health, Mass General Institute for Neurodegenerative Diseases (MIND), Department of Neurology, Massachusetts General Hospital, Harvard Medical School, Charlestown, MA, USA. 
Received: 22 November 2020 Accepted: 9 June 2021 Published online: 20 July 2021

\section{References}

1. Murabito JM, Yuan R, Lunetta KL. The search for longevity and healthy aging genes: insights from epidemiological studies and samples of longlived individuals. J Gerontol A Biol Sci Med Sci. 2012;67(5):470-9. https://doi. org/10.1093/gerona/gls089.

2. Joshi PK, Pirastu N, Kentistou KA, Fischer K, Hofer E, Schraut KE, et al. Genome-wide meta-analysis associates HLA-DQA1/DRB1 and LPA and lifestyle factors with human longevity. Nat Commun. 2017;8(1):910. https:// doi.org/10.1038/s41467-017-00934-5.

3. Deelen J, Evans DS, Arking DE, Tesi N, Nygaard M, Liu X, et al. A metaanalysis of genome-wide association studies identifies multiple longevity genes. Nat Commun. 2019;10(1):3669. https://doi.org/10.1038/s41467-019-11 558-2.

4. Lear SA, Hu W, Rangarajan S, Gasevic D, Leong D, labal R, et al. The effect of physical activity on mortality and cardiovascular disease in 130000 people from 17 high-income, middle-income, and low-income countries: the PURE study. Lancet. 2017;390:2643-54.

5. Westendorp RG, van Heemst D, Rozing MP, Frölich M, Mooijaart SP, Blauw GJ, et al. Nonagenarian siblings and their offspring display lower risk of mortality and morbidity than sporadic nonagenarians: the Leiden Longevity Study. J Am Geriatr Soc. 2009;57(9):1634-7. https://doi.org/10.1111/j.15325415.2009.02381.x

6. Emdin CA, Khera AV, Kathiresan S. Mendelian randomization. Jama. 2017; 318(19):1925-6. https://doi.org/10.1001/jama.2017.17219.

7. Liu Z, Burgess S, Wang Z, Deng W, Chu X, Cai J, et al. Associations of triglyceride levels with longevity and frailty: a Mendelian randomization analysis. Sci Rep. 2017;7(1):41579. https://doi.org/10.1038/srep41579.

8. Postmus I, Deelen J, Sedaghat S, Trompet S, de Craen AJ, Heijmans BT, et al. LDL cholesterol still a problem in old age? A Mendelian randomization study. Int J Epidemiol. 2015;44(2):604-12. https://doi.org/10.1093/ije/dyv031.

9. Arsenault BJ, Pelletier W, Kaiser Y, Perrot N, Couture C, Khaw KT, et al. Association of long-term exposure to elevated lipoprotein(a) levels with parental life span, chronic disease-free survival, and mortality risk: a Mendelian randomization analysis. JAMA Netw Open. 2020;3(2):e200129. https://doi.org/10.1001/jamanetworkopen.2020.0129.

10. Turner MC, Vineis $P$, Seleiro E, Dijmarescu M, Balshaw D, Bertollini $R$, et al. EXPOsOMICS: final policy workshop and stakeholder consultation. BMC Public Health. 2018;18(1):260. https://doi.org/10.1186/s12889-018-5160-z.

11. Sudlow C, Gallacher J, Allen N, Beral V, Burton P, Danesh J, et al. UK biobank: an open access resource for identifying the causes of a wide range of complex diseases of middle and old age. PLoS Med. 2015;12(3):e1001779. https://doi.org/10.1371/journal.pmed.1001779.

12. Noyce AJ, Bandres-Ciga S, Kim J, Heilbron K, Kia D, Hemani G, et al. The Parkinson's disease Mendelian randomization research portal. Mov Disord. 2019;34(12):1864-72. https://doi.org/10.1002/mds.27873.

13. Niedzwiecki MM, Walker DI, Vermeulen R, Chadeau-Hyam M, Jones DP, Miller GW. The exposome: molecules to populations. Annu Rev Pharmacol Toxicol. 2019;59(1):107-27. https://doi.org/10.1146/annurev-pharmtox-01081 8-021315.

14. Pierce $B L$, Ahsan $H$, Vanderweele TJ. Power and instrument strength requirements for Mendelian randomization studies using multiple genetic variants. Int J Epidemiol. 2011;40(3):740-52. https://doi.org/10.1093/ije/dyq1 51.

15. Burgess S. Sample size and power calculations in Mendelian randomization with a single instrumental variable and a binary outcome. Int J Epidemiol. 2014;43(3):922-9. https://doi.org/10.1093/ije/dyu005.

16. Bowden J, Davey Smith G, Haycock PC, Burgess S. Consistent estimation in Mendelian randomization with some invalid instruments using a weighted median estimator. Genet Epidemiol. 2016;40(4):304-14. https://doi.org/10.1 002/gepi.21965.

17. Bowden J, Davey Smith G, Burgess S. Mendelian randomization with invalid instruments: effect estimation and bias detection through Egger regression. Int J Epidemiol. 2015;44(2):512-25. https://doi.org/10.1093/ije/dyv080.

18. Verbanck M, Chen CY, Neale B, Do R. Detection of widespread horizontal pleiotropy in causal relationships inferred from Mendelian randomization between complex traits and diseases. Nat Genet. 2018;50(5):693-8. https:// doi.org/10.1038/s41588-018-0099-7.
19. Hemani G, Zheng J, Elsworth B, Wade KH, Haberland V, Baird D, et al. The MR-Base platform supports systematic causal inference across the human phenome. eLife. 2018;7. https://doi.org/10.7554/ elife.34408.

20. Willer CJ, Schmidt EM, Sengupta S, Peloso GM, Gustafsson S, Kanoni S, et al. Discovery and refinement of loci associated with lipid levels. Nat Genet. 2013;45(11):1274-83. https://doi.org/10.1038/ng.2797.

21. van Oort S, Beulens JWJ, van Ballegooijen AJ, Burgess S, Larsson SC. Cardiovascular risk factors and lifestyle behaviours in relation to longevity: a Mendelian randomization study. J Intern Med. 2021;289(2):232-43. https:// doi.org/10.1111/joim.13196.

22. Andersen SL, Sebastiani P, Dworkis DA, Feldman L, Perls TT. Health span approximates life span among many supercentenarians: compression of morbidity at the approximate limit of life span. J Gerontol A Biol Sci Med Sci. 2012;67(4):395-405. https://doi.org/10.1093/gerona/glr223.

23. Newman AB, Glynn NW, Taylor CA, Sebastiani P, Perls TT, Mayeux R, et al. Health and function of participants in the Long Life Family Study: a comparison with other cohorts. Aging (Albany NY). 2011;3(1):63-76. https:// doi.org/10.18632/aging.100242.

24. López-Otín C, Galluzzi L, Freije JMP, Madeo F, Kroemer G. Metabolic control of longevity. Cell. 2016;166(4):802-21. https://doi.org/10.1016/j.cell.2016.07. 031.

25. Sala ML, Röell B, van der Bijl N, van der Grond J, de Craen AJ, Slagboom EP, et al. Genetically determined prospect to become longlived is associated with less abdominal fat and in particular less abdominal visceral fat in men. Age Ageing. 2015:44(4):713-7. https://doi. org/10.1093/ageing/afv063.

26. Voight BF, Peloso GM, Orho-Melander M, Frikke-Schmidt R, Barbalic $M$, Jensen MK, et al. Plasma HDL cholesterol and risk of myocardial infarction: a mendelian randomisation study. Lancet. 2012;380:57280

27. Do R, Willer CJ, Schmidt EM, Sengupta S, Gao C, Peloso GM, et al. Common variants associated with plasma triglycerides and risk for coronary artery disease. Nat Genet. 2013;45(11):1345-52. https://doi. org/10.1038/ng.2795.

28. Wang J, Shi L, Zou Y, Tang J, Cai J, Wei Y, et al. Positive association of familial longevity with the moderate-high HDL-C concentration in Bama Aging Study. Aging. 2018;10(11):3528-40. https://doi.org/10.18632/a ging.101663.

29. Sun YQ, Burgess S, Staley JR, Wood AM, Bell S, Kaptoge SK, et al. Body mass index and all cause mortality in HUNT and UK Biobank studies: linear and non-linear mendelian randomisation analyses. BMJ. 2019;364: 11042.

30. Global BMIMC, Di Angelantonio E, Bhupathiraju Sh N, Wormser D, Gao P, Kaptoge $\mathrm{S}$, et al. Body-mass index and all-cause mortality: individualparticipant-data meta-analysis of 239 prospective studies in four continents. Lancet. 2016:388:776-86.

31. Dale CE, Fatemifar G, Palmer TM, White J, Prieto-Merino D, Zabaneh D, et al. Causal associations of adiposity and body fat distribution with coronary heart disease, stroke subtypes, and type 2 diabetes mellitus: a Mendelian randomization analysis. Circulation. 2017;135(24):2373-88. https://doi.org/1 0.1161/CIRCULATIONAHA.116.026560.

32. NCD Risk Factor Collaboration. A century of trends in adult human height eLife. 2016;5:e13410. https://doi.org/10.7554/eLife.13410.

33. Samaras $\Pi$. How height is related to our health and longevity: a review. Nutr Health. 2012;21(4):247-61. https://doi.org/10.1177/02601 06013510996.

34. Tanisawa K, Hirose N, Arai Y, Shimokata H, Yamada Y, Kawai H, et al. Inverse association between height-increasing alleles and extreme longevity in Japanese women. J Gerontol A Biol Sci Med Sci. 2018;73(5):588-95. https:// doi.org/10.1093/gerona/glx155

35. He Q, Morris BJ, Grove JS, Petrovitch H, Ross W, Masaki KH, et al. Shorter men live longer: association of height with longevity and FOXO3 genotype in American men of Japanese ancestry. PLoS One. 2014;9(5):e94385. https:// doi.org/10.1371/journal.pone.0094385.

36. Jasilionis D, Shkolnikov VM. Longevity and education: a demographic perspective. Gerontology. 2016;62(3):253-62. https://doi.org/10.1159/00043 8901.

37. Kaplan RM, Howard VJ, Safford MM, Howard G. Educational attainment and longevity: results from the REGARDS U.S. national cohort study of blacks and whites. Ann Epidemiol. 2015;25:323-8. 
38. Permanyer I, Spijker J, Blanes A, Renteria E. Longevity and lifespan variation by educational attainment in Spain: 1960-2015. Demography. 2018;55(6): 2045-70. https://doi.org/10.1007/s13524-018-0718-z.

39. Leong DP, Joseph PG, McKee M, Anand SS, Teo KK, Schwalm JD, et al. Reducing the global burden of cardiovascular disease, part 2: prevention and treatment of cardiovascular disease. Circ Res. 2017;121(6):695-710. https://doi.org/10.1161/CIRCRESAHA.117.311849.

40. Yu JT, Xu W, Tan CC, Andrieu S, Suckling J, Evangelou E, et al. Evidencebased prevention of Alzheimer's disease: systematic review and metaanalysis of 243 observational prospective studies and 153 randomised controlled trials. J Neurol Neurosurg Psychiatry. 2020;91(11):1201-9. https:// doi.org/10.1136/jnnp-2019-321913.

\section{Publisher's Note}

Springer Nature remains neutral with regard to jurisdictional claims in published maps and institutional affiliations.

Ready to submit your research? Choose BMC and benefit from:

- fast, convenient online submission

- thorough peer review by experienced researchers in your field

- rapid publication on acceptance

- support for research data, including large and complex data types

- gold Open Access which fosters wider collaboration and increased citations

- maximum visibility for your research: over $100 \mathrm{M}$ website views per year

At $\mathrm{BMC}$, research is always in progress.

Learn more biomedcentral.com/submissions 